Rev Invest Clin. 2019;71:365-8

\title{
COULD NEUTROPHIL EXTRACELLULAR TraPs BE THE NEW PROGNOSTIC MARKERS OF CANCER?
}

Mónica M. Rivera-Franco ${ }^{1,2 *}$, Eucario León-RodríGueZ², and María de la L. SeVILla-Gonzáleż

${ }^{1}$ Section of Graduate and Research Studies, Escuela Superior de Medicina, Instituto Politécnico Nacional, Mexicódity; 2Department of Hematology and Oncology, Instituto Nacional de Ciencias Médicas y Nutrición Salvador Zubirán Mexico City, Mexico

\begin{abstract}
\end{abstract}
Neutrophil extracellular traps (NETs) were described more than one decade ago, but recently, the interest in these structures has increased due to their involvement in cancer progression, cancer-related thrombosis, and development of metastasis. This protumoral role of NETs strengthens their potential as new prognostic markers of cancer. (REV INVEST CLIN. 2019;71:365-8)

Key words: Neutrophil extracellular traps. Cancer. Metastasis.

\section{INTRODUCTION}

Neutrophil extracellular traps (NETs), which are threedimensional structures containing networks of extracellular fibers, made up of chromatin and neutrophil proteins including peptides with antimicrobial properties, were described to kill bacteria more than one decade ago by Brinkmann et al. ${ }^{1}$. These complex meshworks formed by chromatin and different proteins such as myeloperoxidase (MPO), neutrophil elastase (NE), leukocyte proteinase 3 , cathepsin G, lactoferrin, lysozyme $C$, neutrophil defensins, among others, were afterward associated with lung diseases, many
*Corresponding author:

Mónica M. Rivera-Franco

E-mail:mm.riverafranco@gmail.com chronic inflammatory processes such as autoimmune and auto-inflammatory diseases, cardiovascular illnesses, and even with preeclampsia and infertility ${ }^{2,3}$. At the beginning, the formation of NETs, calledNETosis, was described as a type of cellular deatis however, two mechanisms of NETs release have been observed, one requiring the rupture of the cytoplasmic membrane of the neutrophil (lytic) and the other one not requiring the lytic suicide of the cell $(v i t a l)$. Overall, this process typically relies on common neutrophil enzymes such as MPO and NE and on the production of reactive oxygen species ${ }^{2}$, as well as on the neutrophil release of nuclear chromatin involving 
the citrullinated histone $\mathrm{H} 3$. Despite inflammation potentially being the primary cause of the formation of NETs, many factors, for example chemokines, can also promote this phenomenon. Recently, the interest in NETs has increased due to their involvement in a multitude of pathophysiological processes. For instance, they have been described to be related with cancer progression, cancer-related thrombosis, and the development of metastasis.

\section{NETS AND CANCER}

Worldwide, cancer is a leading cause of morbi-mortality. Despite advances within the oncology field focusing on diagnosis and prognosis, metastasis is one of the main causes of cancer-related death and its prevention remains to be a major clinical challenge. On the other hand, neutrophils are the most abundant granulocytes in humans, accounting more than half of the circulating leukocytes. Many studies have associated these cells with cancer as they have been described to function as independent markers of prognosis, but more importantly, it is known that mutations in normal cells are not the exclusive factors initiating and sustaining tumor progression. The majority of the components in NETs have the potential to stimulate tumor growth, promote angiogenesis, and develop metastasis; this is acknowledged as most of the associated proteins have been studied prior the description of these complex structures ${ }^{2}$.

The first study reporting an association between NETs and cancer was published in 2013, suggesting that patients with Ewing sarcoma had a poorer prognosis when intratumoral NETs were present ${ }^{4}$. Later, a murine model demonstrated that more neutrophils were recruited in the context of breast cancer cells that metastasized. Furthermore, these breast cancer metastatic cells stimulated the formation of NETs, which were effectively inhibited by the injection of DNAse $I^{5}$. These findings were also corroborated in an in vitro model. Triple-negative breast cancer cells were isolated from a genetically modified mice model and from patient samples, inducing NETs when cocultured with both, mice and healthy human volunteer neutrophils ${ }^{5}$. Although metastasis-supporting NETs might form in the absence of infection, Cools-Lartigue et al. ${ }^{6}$ used a murine model (injected with lung cancer cell lines) of systemic sepsis caused by cecal ligation and puncture to develop macrometastasis that was afterward attenuated by the administration of inhibitors of the formation oE NETs, such as DNAse I and NE inhibitor. The authors also reported that NETs were sufficient to increase the adhesion of tumor cells within the hepatic sinusoids and thus considered as a neutrophil-dependent adhesive mechanism ${ }^{6}$. This group also investigated potential mechanisms through which NETs were involved in cancer progression after interacting with circulating tumor cells, describing the role of $\beta 1$ integrin in mediating the adhesion of cancer cells to NETs as this protein was expressed in both, NET $\$$ and tumor cells.

As the adhesive properties of NETs enable pathogen and platelet binding, this could also facilitate tumor adhesion and consequently metastasis ${ }^{2}$. Overall, it is currently known that tumor cells and their environment can prime neutrophils to form NETs leading to tumor growth ${ }^{2}$. Moreover, under inflammatorysconditions, neutrophils promote the trapping of circulating tumor cells, and thus, the potential role of NETS Sin this process is suggested. In addition, tumor recturrence can occur after surgical stress ${ }^{7}$. Ischemia and rêperfusion injury as a consequence of surgery to treat colorectal liver metastasis can generate NETs rine models and accordingly, high levels of NETS, detected in human serum samples of patients with metastatic colorectal cancer undergoing a major liver resection, were associated with a higher incidence of recurrence ${ }^{7}$.

Nonetheless, most of the studies evaluating NETs have been performed using in vitro or murine cell lines, and instead of measuring circulating NETs, some studies have induced their formation throughout neutrophil stimulation. To date, only few studies have evaluated complexes directly from serum samples or tissue from cancer patients, and more dmportantly, due to the current paucity of specifie antibodies directed toward exclusive epitopes in NETs, some researches have only demonstrated the presence of neutrophils, components of NETs, orextracellular DNA, and thus, it seems that there is no standard assay to evaluate NETs. However, the most specific complex to detect NETs includes DNA or chromatin and neutrophil proteins (NE or MPO), demonstrated by immunofluorescence or sandwich ELISA. 
Figure 1. Roles of NETs in cancer. NETs act as a barrier between the tumor and the cells from the immune system (step 1); NETs promote the tumor microenvironment (step 2); NETs associated with thrombosis (step 3); NETs capture cancer cells and promote migration and metastasis (step 4$)^{8}$.
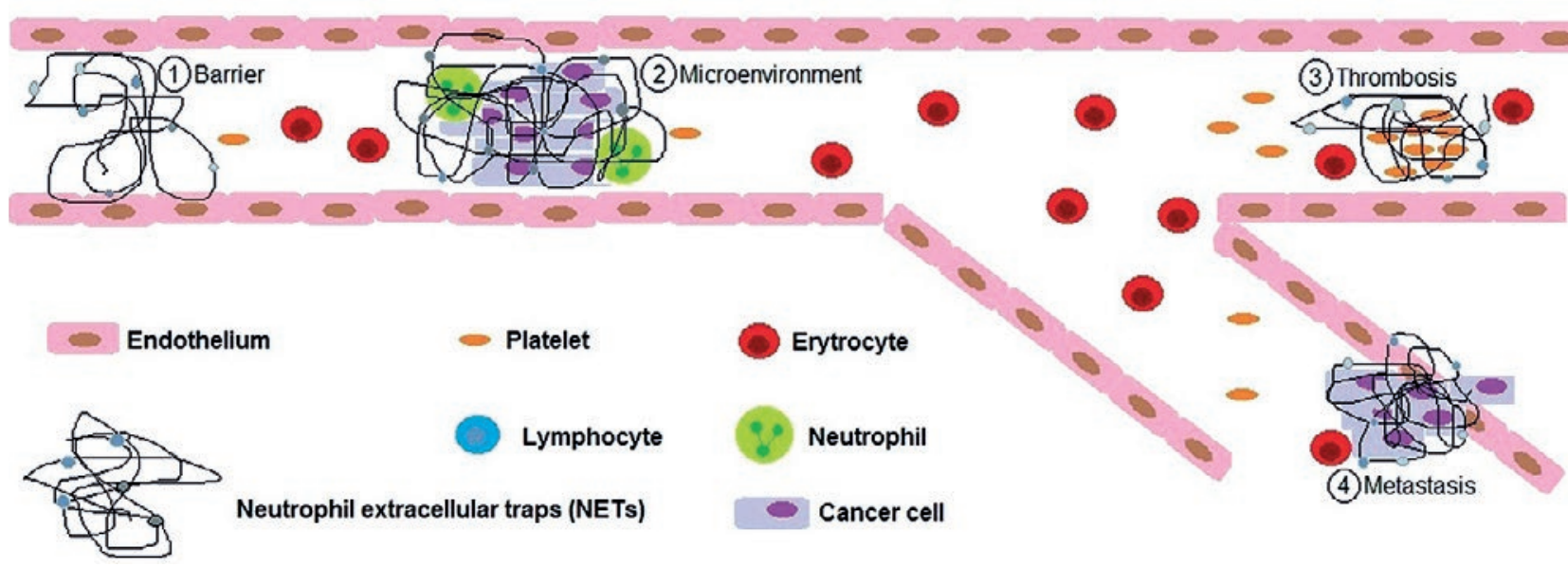

(4) Metastasis

Cancer cell

On the other hand, the existence of different tumor-infiltrating immune cell populations including leukocytes, lymphocytes, macrophages, dendritic cells, and mastocytes has been well documented. In this context, there are in vitro studies demonstrating a relationship between NETs and other cells from the immune system. NETs activate T Iymphocytes and dendritic cells, and also, they intervene in the recruitment of dendritic cells within the tumor environment and the formation of a barrier between the immune cells and the tumor, as well as the inhibition of the function of the former ${ }^{8}$.

In addition, cancer has been associated with thrombosis, which is an important cause of death among this population of patients. Although the exact mechanism remains unknown, NETs have been described to trigger thrombogenicity and this phenomenon could be explained by neutrophils themselves and histone release but also due to the platelet trapping by NETs and the initiation of the formation of the thrombus ${ }^{2,8}$. In addition, some elements of NETs might induce platelet activation, accumulation of erythrocytes, and the release of von Willebrand factor, which is known as one of the main components of thrombus ${ }^{8}$. In vitro studies have shown that venous thrombi could be significantly reduced when NETs were targeted with NE inhibitor and DNAse I. Furthermore, data suggest that leukemia and cancer cells produced factors contributing to the formation of NETs, which were at the same time a predisposition for thrombosis ${ }^{2,8}$. The different currently accepted roles of NETs in cancer are summarized in figure 1 .

\section{CONCLUSION}

Although evidence suggests that neutrophils alone can facilitate cancer progression, additionally to one of their main functions as the first line of defense during infections, recently, the formation of NETs and their interaction with cancer cells and other factors of the immune system appear to be mandatory to contribute to the development of metastasis. To date, the role of NETs in many types of cancer has not been completely defined as studies have described this interaction in the setting of infections, surgical stress, or specific populations of $\frac{\complement}{\sigma} a n c e r$ cells, and unfortunately, many reports have ben limited because they failed to detect real NETS, as there is paucity of a standardized technique Thus, further studies are required to comprehend the whole nature and mechanism of NETs to properly intervene within their formation to potentially improve clinical outcomes. However, the already described protumoral role of these complex structures strengthens their potential as a new prognostic marker of cancer. 


\section{REFERENCES}

1. Brinkmann V, Reichard U, Goosmann C, Fauler B, Uhlemann Y, Weiss DS, et al. Neutrophil extracellular traps kill bacteria. Science. 2004;303:1532-5.

2. Erpenbeck L, Schön MP. Neutrophil extracellular traps: protagonists of cancer progression? Oncogene. 2017;36:2483-90.

3. Brinkmann V. Neutrophil extracellular traps in the second decade. J Innate Immun. 2018;10:414-21.

4. Berger-Achituv S, Brinkmann V, Abed UA, Kühn LI, Ben-Ezra J, Elhasid R, et al. A proposed role for neutrophil extracellular traps in cancer immunoediting. Front Immunol. 2013;4:48.
5. Park J, Wysocki RW, Amoozgar Z, Maiorino L, Fein MR Jorns ], et al. Cancer cells induce metastasis-supporting neutrophil extracellular DNA traps. Sci Transl Med. 2016;8:361ra138.

6. Cools-Lartigue J, Spicer J, McDonald B, Gowing S, Chow S, Giannias B, et al. Neutrophil extracellular traps sequester circulating tumor cells and promote metastasis. J Clin Invest. 2013 123:3446-58

7. Tohme S, Yazdani HO, Al-Khafaji AB, Chidi AP, Loughran P, Mowen $\mathrm{K}$, et al. Neutrophil extracellular traps promote the development and progression of liver metastases after surgical stress. Cancer Res. 2016;76:1367-80.

8. Garley M, Jabłońska E, Dąbrowska D. NETs in cancer-Tumour Biol. 2016;37:14355-61. 